\title{
Outcomes and risk factors identification in urgent lung transplantation: a multicentric study
}

\author{
Marco Schiavon ${ }^{1}$, Giulio Faggi ${ }^{1}$, Lorenzo Rosso ${ }^{2}$, Luca Luzzi $^{3}$, Giovanni Maria Comacchio ${ }^{1}$, \\ Dario Gregori ${ }^{4}$, Mario Nosotti ${ }^{2}$, Francesco Damarco ${ }^{2}$, Andrea Dell'Amore ${ }^{1}$, David Bennet ${ }^{3}$, \\ Antonella Fossi ${ }^{3}$, Piero Paladini ${ }^{3}$, Luigi Santambrogio ${ }^{2}$, Federico Rea ${ }^{1}$
}

${ }^{1}$ Thoracic Surgery and Lung Transplantation Unit, University-Hospital of Padova, Padova, Italy; ${ }^{2}$ Thoracic surgery and Lung Transplantation Unit, Fondazione IRCCS Ca' Granda, Ospedale Maggiore Policlinico, Milano, Italy; ${ }^{3}$ Lung Transplantation Unit, Department of Medical, Surgical and Neurosciences, University of Siena, Siena, Italy; ${ }^{4}$ Statistic Unit, Department of Cardiac, Thoracic, Vascular Unit and Public Health, University of Padova, Padova, Italy

Contributions: (I) Conception and design: M Schiavon, G Faggi; (II) Administrative support: D Gregori; (III) Provision of study materials or patients: M Schiavon, F Rea, L Rosso, M Nosotti, L Luzzi, P Paladini; (IV) Collection and assembly of data: M Schiavon, G Faggi, L Rosso, F Damarco, L Luzzi, D Bennet; (V) Data analysis and interpretation: D Gregori, M Schiavon, G Faggi; (VI) Manuscript writing: All authors; (VII) Final approval of manuscript: All authors.

Correspondence to: Marco Schiavon, MD, PhD. Department of Cardio-Thoracic and Vascular Sciences, Division of Thoracic Surgery, University of Padova, Via Giustiniani, 2 - 35128 Padova, Italy. Email: marco.schiavon@unipd.it.

Background: In rapidly lung deteriorating patients, urgent lung transplantation (ULT) seems the only definitive therapy. Few publications on this topic report conflicting results, putting a word of caution about ULT programs.

Methods: A national ULT program was introduced in 2010: patients on mechanical support may be transplanted with the first available graft. We reviewed the experience of three national center, focusing on post-operative outcomes after ULT.

Results: Ten patients (17.5\%) died awaiting transplantation, while 47 underwent LT with a median urgent waiting list time of 6 days. Pre-operatively, $4.3 \%$ of patients were supported only by mechanical ventilation (MV), 55.3\% by extracorporeal membrane oxygenation (ECMO) and the remaining $40.4 \%$ by both. The main indication was cystic fibrosis (64\%). Median recipient lung allocation score was 72 . In-hospital mortality was $19 \%$. MV and ECMO median duration of 7 and 3 days, respectively while intensive care unit (ICU) and hospital stay were 20 and 46 days, respectively. At long-term, 1- and 3-year survival rate were 74\% and $70 \%$, respectively. Highly impact risk factors for in-hospital mortality were both presence and duration of preoperative veno-arterial ECMO and pre-transplant C-reactive protein level.

Conclusions: ULT program allows transplantation in a significant percentage of patients with acceptable results. Pre-operative recipient selection is mandatory to improve clinical outcomes.

Keywords: Lung transplantation (LT); extracorporeal membrane oxygenation (ECMO); C-reactive protein (CRP); lung allocation score

Submitted Jun 03, 2019. Accepted for publication Aug 26, 2019.

doi: $10.21037 /$ jtd.2019.10.55

View this article at: http://dx.doi.org/10.21037/jtd.2019.10.55

\section{Introduction}

Nowadays, lung transplantation (LT) has become a widely used life-saving procedure for patients suffering from endstage lung diseases. Nonetheless, the increasing number of LT still does not match the number of patients on the waiting list (WL), who are exposed to such a worsening of their clinical conditions requiring supportive therapy as mechanical ventilation (MV) or extracorporeal membrane 
oxygenation (ECMO). Despite their time-sparing capacity, these strategies can only be temporary and do not represent a definitive solution, only achievable with transplantation.

In the last decade, in addition to strategies to expand the donor pool [extended criteria donors (ECD) and ex-vivo lung perfusion (EVLP)], for patients with a high WL mortality, several approaches [i.e., lung allocation score (LAS) and urgent lung transplantation (ULT)] to increase the LT effectiveness have been developed in the transplant community (1-3).

In particular, in Italy a national ULT program began in 2010 and an early report of its application was published, with encouraging results (4). The two goals to consider when approaching ULT are the reduction of WL time and mortality assuring at the same time a postoperative survival comparable to elective procedures. Indeed, we recently demonstrated that the application of ULT in a singlecentre experience, despite a worse postoperative mortality compared to national results, confirmed the first goal of the program; in addition, previous unrecognized preoperative recipient risk factors for early fatal outcome were identified (5). Both these studies presented a weakness point in the number of patients involved and the short period analysed especially in the first one. To overcome these limitations, a multicentre study was performed to better understand the reliability of this program and eventually confirm our previous findings.

\section{Methods}

This is a retrospective multicenter analysis among patients listed for LT in three institutions (Milano, Siena and Padova) between January 2011 and July 2016. This study was approved by every single Institutional review board (protocol number 0027736) and performed in accordance with accepted ethical standards. Informed consent was obtained before using patients' data.

According to the Italian Urgent Lung Transplant program, inclusion or exclusion criteria were defined to minimize the percentage of graft waste.

Urgency criteria are defined as follows:

* Patients on the WL dependent on MV and/or extracorporeal membrane oxygenator (except for Extracorporeal Carbon Dioxide Removal device);

* Age $\leq 50$ years old;

* Patients may be transplanted on an emergency basis with the first available graft in the country;

* Previous LT WL.
Patients with sepsis, multiorgan failure, hemorrhagic shock or neurologic impairment are excluded from the urgency program. Retransplantation is not considered an exclusion criterion. Since 1 week of priority is allowed and may be renewed only twice. The longest urgent WL time does not exceed 3 weeks.

LAS (6) was retrospectively calculated to define recipient clinical condition. Donor's characteristics were assessed according to Oto score (7). Extended criteria donors were considered grafts with more than 55 years, a smoking history higher than 20 packs/year and a $\mathrm{PaO}_{2} / \mathrm{FiO}_{2}$ lower than $300 \mathrm{mmHg}$ after recruitment maneuvers. Preoperative recipient monitoring included a full evaluation of respiratory, hemodynamic, hematologic, renal and liver function. Pulmonary hypertension (PH, mean pulmonary artery pressure $\geq 25 \mathrm{mmHg}$ ) was diagnosed with right heart catheterization, in accordance with 2015 European Society of Cardiology/European Resuscitation Council (ESC/ERC) Guidelines (8). We recorded comprehensive intra-operative data, including use of ECMO and cold ischemic times for all transplanted patients. Postoperative data collected for all recipients included primary graft dysfunction (PGD) scores at time $0,24,48$, and $72 \mathrm{~h}(9)$, time on MV after surgery, length of intensive care unit (ICU) stay and in-hospital stay, in hospital mortality and morbidity. All transplanted patients were followed up for incidence of acute rejection at one month with trans-bronchial biopsy (10), for pulmonary function with measurement of 1 -year $\mathrm{FEV}_{1} \%$ and for postoperative survival at 1 - and 3 -year periods.

\section{ECMO management}

Indication for ECMO support as a bridge for LT was an acute respiratory insufficiency with severe hypoxia and or hypercarbia with $\mathrm{pH}$ less than 7.25 and/or the inability to maintain adequate gas exchange despite aggressive MV. The veno-venous (VV) ECMO configuration was the preferred strategy for patients with respiratory failure with stable hemodynamic while in subjects with concomitant compromised cardiac function or with severe pulmonary artery hypertension (primary or secondary) or with hemodynamic instability, a veno-arterial (VA) configuration was adopted. Peripheral ECMO was routinely performed through percutaneous or surgical way on the femoral vessels, while trans-esophageal echocardiography was used to confirm the correct placement of the venous cannula into the right atrium just in front to the tricuspid orifice. At the time of LT the preoperative ECMO setting was maintained 
Table 1 Preoperative characteristics of the study population

\begin{tabular}{|c|c|}
\hline Variable & ULT $(n=47)$ \\
\hline Age (years) & $30[21-40]$ \\
\hline Sex (male/female) & $13 / 34$ \\
\hline $\mathrm{BMI}, \mathrm{kg} / \mathrm{m}^{2}$ & $19(17.5-21.5)$ \\
\hline \multicolumn{2}{|l|}{ Diagnosis } \\
\hline $\mathrm{CF}$ & $30[64]$ \\
\hline IPF & 9 [19] \\
\hline Retransplantation in BOS & $1[2]$ \\
\hline Other & 7 [15] \\
\hline $\mathrm{PH}$ & $24[51]$ \\
\hline PsA colonization & $21[45]$ \\
\hline Waiting time on regular list (days) & $52[5-264]$ \\
\hline Waiting time on UWL (days) & $6[3-9]$ \\
\hline LAS at transplantation & 72 [45-83] \\
\hline \multicolumn{2}{|l|}{ Type of support } \\
\hline MV & $21[45]$ \\
\hline ECMO & $45[96]$ \\
\hline VV & $42[93]$ \\
\hline VA & $3[7]$ \\
\hline \multicolumn{2}{|l|}{ Patients transfused } \\
\hline RBC & 40 [85] \\
\hline FFP & $7[15]$ \\
\hline PTL & $0[0]$ \\
\hline Fibrinogen & 1 [2] \\
\hline RBC transfusion (units) & $4[2-5]$ \\
\hline
\end{tabular}

Data are reported as median $\left(1^{\circ}-3^{\circ}\right.$ quartile) for continuous and as number (percentage) for categorical variables. ULT, urgent lung transplantation; BMI, body mass index; CF, cystic fibrosis; IPF, idiopathic pulmonary fibrosis; BOS, bronchiolitis obliterans syndrome; $\mathrm{PH}$, pulmonary hypertension (diagnosed trough right heart catheterization, see text); PsA, pseudomonas aeruginosa; UWL, urgent waiting list; LAS, lung allocation score; MV, mechanical ventilation; ECMO, extracorporeal membrane oxygenation; VV, veno-venous; VA, veno-arterial; RBC, packed red blood cells; FFP, fresh frozen plasma; PTL, platelets.

as intraoperative support with VV ECMO setting changed to VA central cannulation (aorto-right atrium) in case of intraoperative deterioration of the hemodynamic or respiratory parameters. At the end of the operation whenever possible the ECMO support was gradually reduced and stopped. In selected cases, ECMO support was prolonged in the post-operative period: marginal donors, long ischemic time $(>7 \mathrm{~h})$, high risk recipient [primitive $\mathrm{PH}$, idiopathic pulmonary fibrosis (IPF) with severe $\mathrm{PH}$, right ventricular impairment], lobar LT, severe pulmonary artery hypertension after reperfusion ( $>2 / 3$ the systemic pressure), high requirement of inotropes, signs of early reperfusion lung injury with rapid worsening of the respiratory and hemodynamic parameters, need for aggressive ventilation to maintain acceptable gas exchange.

\section{Statistical analysis}

Basic exploratory data analysis has been performed on the sample and reported using median (I-III quartile) for continuous variables and percentages (absolute numbers) for categorical variables, whenever appropriate. Chi-square tests or Wilcoxon signed rank test have been used for evaluating significance of differences in factors distribution. Survival curves were estimated using Kaplan-Meier estimator with $95 \%$ confidence bands. Comparison among curves was based on log-rank test. Effect of covariates on survival were based to a Cox proportional hazard model and expressed as hazard ratio (HR) with corresponding $95 \%$ confidence interval (CI). Non-linear effects of continuous covariates on survival were estimated using restricted cubic splines (11). Significance of findings was evaluated using Akaike Information Criterion and its statistical significance (for inclusion in the model of any given covariate) has been computed. Probability values of $\mathrm{P}<0.05$ was in general accepted as significant. All analyses were performed using the R System (12) and the rms libraries (13).

\section{Results}

\section{Preoperative characteristics (Table 1)}

In the study period, 57 patients ( 13 males, median age 30 years) were included in the urgent waiting list (UWL); among them, 10 (17.5\%) died in WL while 47 (82.5\%) underwent LT (44 bilateral).

Considering the transplanted subjects, cystic fibrosis accounted for the most of the cases (30 patients, 64\%); only one case of retransplantation was recorded for chronic rejection 2 years after primary LT for cystic fibrosis. Secondary PH was diagnosed in 24 (51\%) subjects. Pseudomonas aeruginosa colonization was found in 21 patients (45\%), and six of these strains were multi-drug 
Table 2 Donors' characteristics

\begin{tabular}{lc}
\hline Variable & ULT $(\mathrm{n}=47)$ \\
\hline Age (years) & $45[34-50]$ \\
Oto score & $4(2.5-6.5)$ \\
Cold ischemic time (minutes) & \\
First lung & $345[287-440]$ \\
$\mathrm{Second}$ lung & $520[408-628]$ \\
$\mathrm{PaO}_{2} / \mathrm{FiO}_{2}$ ratio $(\mathrm{mmHg})$ & $445[368-507]$ \\
$\mathrm{ICU}$ (days) & $3[1-4]$ \\
\hline
\end{tabular}

Data are reported as median $\left(1^{\circ}-3^{\circ}\right.$ quartile). ULT, urgent lung transplantation; $\mathrm{PaO}_{2} / \mathrm{FiO}_{2}$, pressure arterial oxygen/fractional inspired oxygen; ICU, intensive care unit.

resistant. All patients were in the elective $\mathrm{WL}$ for 52 days [interquartile range (IQR), 5-264 days], with a median LAS of 38.5 (IQR, 35-41); in UWL, LAS increased to 72 (IQR, 45-83) and waiting time to LT was 6 days (IQR, 3-9 days).

Preoperatively, 45 (96\%) patients were bridged on peripheral ECMO for 6 days (IQR, 4-11.5 days), 42 with VV ECMO. Twenty-one patients were on MV for 4.3 days (IQR, 0-6 days): 19 were on ECMO also (16 VV), while 2 subjects were on MV only. VA ECMO bridge to LT in addition to $M V$ was instituted in 3 cases. All of them presented secondary $\mathrm{PH}$. Forty $(85 \%)$ patients required red blood cell (RBC) transfusion and 7 (15\%) FFP.

Table 2 reported the main donor's characteristics.

Donor's median Oto score was 4 (IQR, 2.5-6.5) and 16 (34\%) were considered ECD, according to one or more marginal criteria, including age (nine donors), low $\mathrm{PaO}_{2} /$ $\mathrm{FiO}_{2}$ (four donors) or smoking history (seven donors). Eight (17\%) grafts underwent EVLP before transplantation, four of them with preservation purposes and four for reconditioning.

\section{Intraoperative results (Table 3)}

Median cold ischemic time was 345 (IQR, 287-440) and 520 (IQR, 408-628) minutes for the first and the second lung, respectively. All the patients were supported with ECMO during LT procedure, 21 (45\%) with VV ECMO. All but three patients (94\%) required RBC, 27 (57\%) FFP, 15 (32\%) platelets; median number of RBC unit used was 8 (IQR, 3-10). One patient died intraoperatively due to massive bleeding $(2 \%)$.
Table 3 Intraoperative results

\begin{tabular}{ll}
\hline Variable & ULT (n=47) \\
\hline Number of BSLT & $94[44]$ \\
Size reduction & $11[23]$ \\
Unilateral & $5[45]$ \\
Bilateral & $6[55]$ \\
ECMO & $47[100]$ \\
VA & $26[55]$ \\
VV & $21[45]$ \\
Patients transfused & \\
RBC & $44[94]$ \\
FFP & $27[57]$ \\
PTL & $15[32]$ \\
Transfusion (units) & \\
RBC & $8[3-10]$ \\
FFP & $3[0-6]$ \\
PTL & $0[0-1]$ \\
Intraoperative death & $1[2]$ \\
\hline
\end{tabular}

Data are reported as median $\left(1^{\circ}-3^{\circ}\right.$ quartile) for continuous and as number (percentage) for categorical variables. ULT, urgent lung transplantation; BSLT, bilateral sequential lung transplantation; ECMO, extracorporeal membrane oxygenation; VA, veno-arterial; VV, veno-venous; RBC, packed red blood cells; FFP, fresh frozen plasma; PTL, platelets.

\section{Postoperative results (Table 4)}

Eleven $(23 \%)$ patients were successfully weaned from extracorporeal support immediately after the procedure, while the remaining 35 (76\%) needed postoperative ECMO (28 VV and $7 \mathrm{VA}$ ) support. Among them, 33 (94\%) were successfully weaned after a median duration of 3 days (IQR, 1-4 days), whilst four of them required prolonged VA ECMO for more than 5 days. MV was continued for 7 days (IQR, 3-24 days), and 23 (50\%) patients needed percutaneous tracheostomy whilst staying in ICU. PGD grade $2-3$ was diagnosed in $54 \%, 63 \%, 46 \%$ and $39 \%$ of subjects at $0,24,48$ and $72 \mathrm{~h}$, respectively. All the patients received postoperative $\mathrm{RBC}$ transfusion with a median value of 9 (IQR, 4-17) units/patient.

In-hospital mortality was $19 \%$ (9 patients), with two subjects still on VA ECMO, on day 7 and 8, respectively. 
Table 4 Postoperative period

\begin{tabular}{|c|c|}
\hline Variable & $\operatorname{ULT}(n=46)$ \\
\hline Postoperative ECMO & 35 [76] \\
\hline VV & $28[80]$ \\
\hline VA & $7[20]$ \\
\hline ECMO duration (days) & $3[1-4]$ \\
\hline MV duration (days) & 7 [3-24] \\
\hline \multicolumn{2}{|l|}{ PGD score grade $2-3$} \\
\hline 0 hours & $25[54]$ \\
\hline 24 hours & 29 [63] \\
\hline 48 hours & $21[46]$ \\
\hline 72 hours & 18 [39] \\
\hline Major complications & 32 [70] \\
\hline CVVH & $18[56]$ \\
\hline Surgical revision & $16[50]$ \\
\hline Other & $5[16]$ \\
\hline In hospital acute cellular rejection & $6[13]$ \\
\hline Tracheostomy & $23[50]$ \\
\hline Infections & $37[80]$ \\
\hline \multicolumn{2}{|l|}{ Patients transfused } \\
\hline $\mathrm{RBC}$ & $46[100]$ \\
\hline FFP & 28 [61] \\
\hline PTL & 18 [39] \\
\hline \multicolumn{2}{|l|}{ Transfusion (units) } \\
\hline $\mathrm{RBC}$ & $9[4-17]$ \\
\hline FFP & $2[0-7]$ \\
\hline PTL & $0[0-1]$ \\
\hline ICU stay (days) & $19[10-31]$ \\
\hline Hospital stay (days) & 45 [32-73] \\
\hline In-hospital mortality & 9 [20] \\
\hline 1-year FEV F $_{0}$ & $70[56-85]$ \\
\hline 1-year survival \% & 74 \\
\hline 3-year survival \% & 70 \\
\hline
\end{tabular}

Data are reported as median $\left(1^{\circ}-3^{\circ}\right.$ quartile) for continuous and as number (percentage) for categorical variables. ECMO, extracorporeal membrane oxygenation; VA, veno-arterial; VV, veno-venous; MV, mechanical ventilation; PGD, primary graft dysfunction; CVVH, continuous veno-venous hemofiltration; RBC, packed red blood cells; FFP, fresh frozen plasma; PTL, platelets; ICU, intensive care unit; $\mathrm{FEV}_{1}$, forced expiratory volume in 1 second.
Postoperative complications burdened 32 (70\%) patients: 18 recipients presented acute kidney injury requiring continuous VV hemofiltration, 16 required surgical revisions, mostly due to hemothorax and five patients suffered main vein thrombosis (caval, femoral or jugular).

Patients were discharged from ICU after 19 days (IQR, 10-31 days), while the overall hospital stay was 45 days (IQR, 32-73 days). Six patients (13\%) presented 30-day acute rejection, successfully treated with steroid pulse therapy. After a median follow-up period of 721 days (IQR, 164-1,097 days), 1 - and 3-year survival was $74.2 \%$ and $69.9 \%$, respectively. Finally, 1-year $\mathrm{FEV}_{1}$ was $70 \%$ (IQR, $56-85 \%)$ of predicted value.

\section{Analysis of risk factors for in-hospital mortality}

According to previous results, we focused on pre-operative donor and recipient characteristics that may affect postoperative mortality.

In this analysis, patients bridged on VA ECMO (HR 4.86 vs. VV, 95\% CI: 0.76-31.06, Figure 1) and days spent on VA ECMO (HR 9.46 over 18 days, 95\% CI: 0.51-173.11, Figure 2) were the two main factors predictive for inhospital mortality. In particular, the risk increases from the first to the fifth day on VA ECMO, thereafter seems to reach a plateau.

Among pre-operative biochemical factors, high C-reactive protein (CRP) levels were strongly linked to in-hospital mortality (HR 29.59), differently from procalcitonin increase (Figure 3). In addition, post-operative mortality was found increased in those patients with LAS higher than 90 (HR 1.25 for every unit above 90, Figure 4).

\section{Discussion}

Worldwide, the amount of LT procedures is still lower than required, and this is mainly due to the limited donor pool. For rapidly worsening recipient, programs like LAS (3) or emergency listing (2) were introduced with the purpose to give them a priority access to LT, reporting promising results, but also giving rise to ethical issues. In fact, the risk of transplanting a "too sick patient" places a word of caution on such programs, and the optimal match between donor and recipient now seems essential, in order not to waste organs (14-16).

We previously investigated how the national priority allocation program worked in Padua Hospital, with encouraging results concerning the access to transplantation 


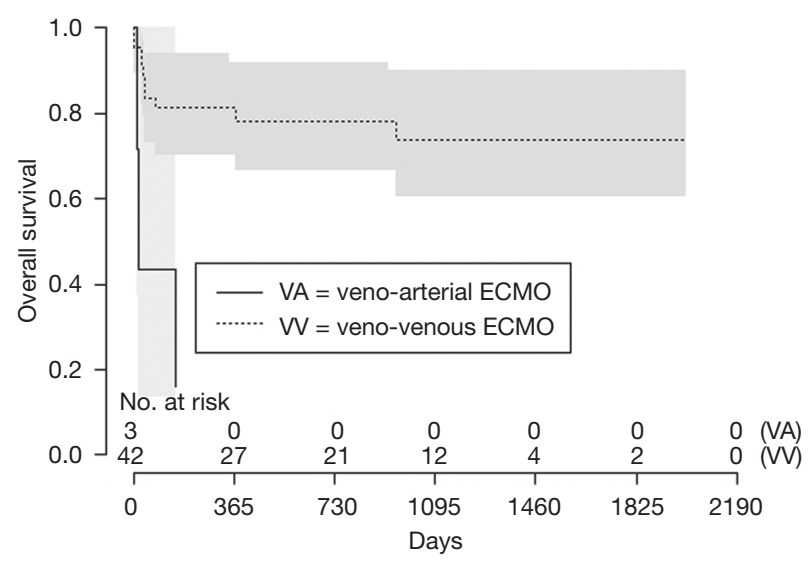

Figure 1 Kaplan-Meyer curves reporting post-transplant survival according to type of pre-operative ECMO support. The presence of veno-arterial (VA) ECMO was associated to a significant increase (HR 4.86) of post-operative mortality compared to veno-venous (VV) ECMO. ECMO, extracorporeal membrane oxygenation; HR, hazard ratio.

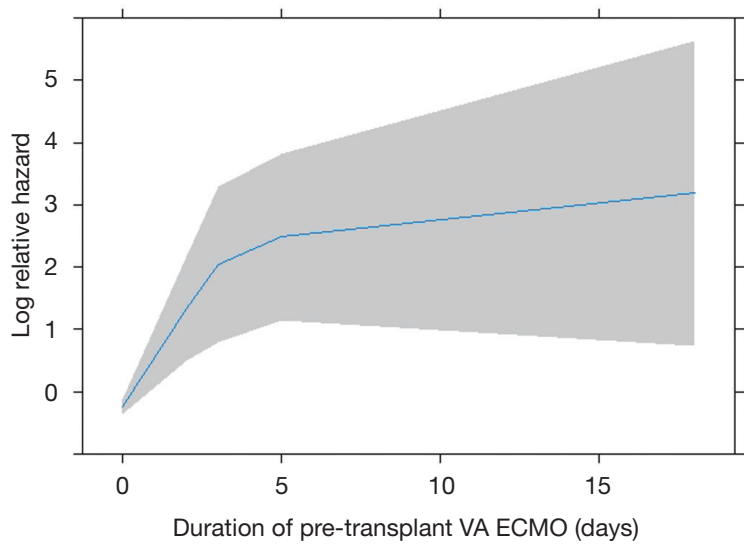

Figure 2 The association of pre-operative VA ECMO support duration and post-operative mortality risk (HR 9.46). The slope of the curve increases from the first to the fifth day on VA ECMO, thereafter seems to reach a plateau.VA, veno-arterial; ECMO, extracorporeal membrane oxygenation; HR, hazard ratio.

and mid-term outcomes (5).

In this present paper, we collected data from 3 main Italian LT centers after 66 months of ULT implementation, and results are likely to support our single centre data. In fact, our multicentre study confirms the utility of an emergency listing program in a country with a shortage of donors.

In Italy the data published from Centro Nazionale
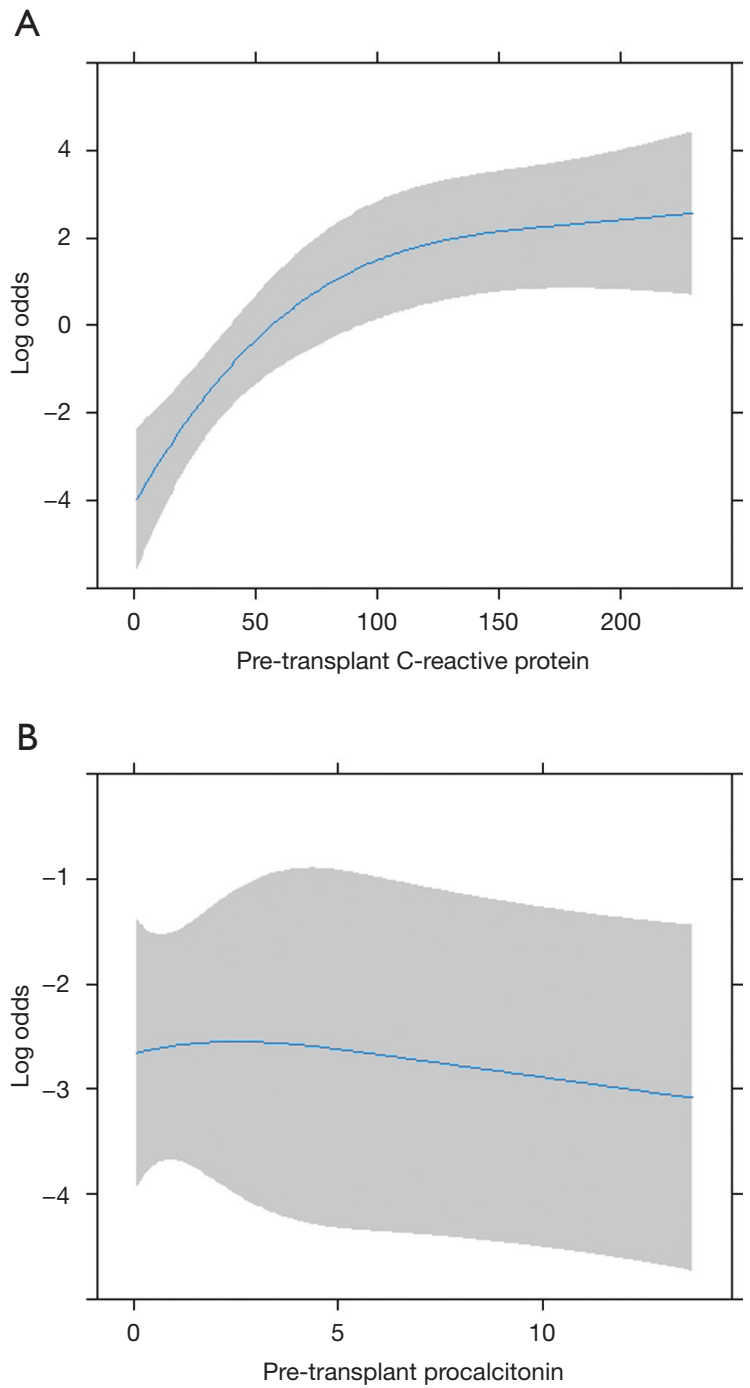

Figure 3 The association between C-reactive protein (CRP) and procalcitonin with in-hospital mortality. Only the increase of the first plasmatic marker value is associated with a higher death risk.

Trapianti (CNTO-REGDON) reported for 2017 a mean WL time for LT of 1.1 years with a mortality of $8.6 \%$ (48 patients out of 556) considering a transplant number attested to 144 procedures.

According to this data, we confirmed that the urgent $W L$ allows transplantation in a short time period to a high percentage of patients with acceptable rate of WL deaths; moreover, given the high complexity of these patients, postoperative complications rate, in-hospital mortality and mid-term survival are also reasonable.

In particular, in-hospital mortality (18\% vs. $37.5 \%)$ and both 1 - and 3 -year survival (74\% and $70 \%$ vs. $57.6 \%$, 


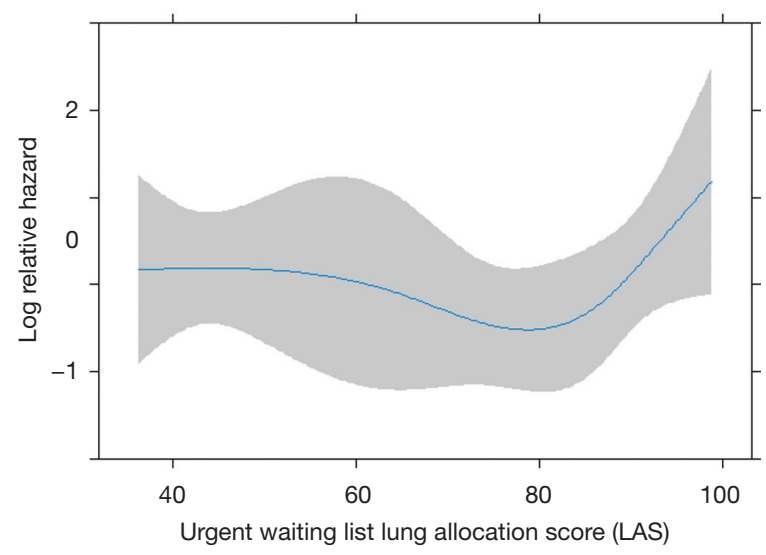

Figure 4 The association of urgent waiting list lung allocation score (LAS) with post-operative mortality. The increased risk is observed in patients with LAS higher than 90 (HR 1.25 for every unit above 90). HR, hazard ratio.

respectively) are better than what we previously reported, while postoperative complications rate and hospital stay are not significantly higher. Notably, after discharge from hospital the number of deaths is limited to 4 in the first year and only 2 more in following 2 years. This could lead to the conclusion that the first postoperative phase is the crucial one, thus probably meaning some corrections to recipient selection is still needed (17). Also compared to the present major studies on emergency transplants (2), our results confirm the usefulness and effectiveness of the Italian program, with post-operative outcomes that compare favourably with those reported by the multicenter French study (18), presenting a higher intraoperative death $(8.4 \%$ vs. $2.1 \%)$ and in-hospital mortality (35.8\% vs. $18 \%)$ and a lower 1-year survival (67.2 \% vs. $74 \%$, respectively). At the same time, the previous work by Boffini $e t$ al. analyzing the initial phase of the Italian national program, showed a comparable post-operative mortality, but assessed at 30 days (4).

According to our results, the identification of preoperative risk factors may be fundamental to improve early post-operative outcomes and to avoid an inappropriate use of lung grafts, included in the analysis of both donor and recipient characteristics. In fact, recent studies pointed out the importance of considering also the quality of the graft, in particular when this is going to be transplanted to a critical recipient or if it is an ECD (19,20). Mulligan and colleagues noted that (21) the use of ECD was associated to reduce 1-year survival, and this decrease was particularly marked in high LAS recipients. Moreover, Sommers and colleagues (22) advocate the use of ECD for stable patients: they do not use ECD for recipients on MV or ECMO.

In our experience, no influence of donor's graft characteristics on the different outcomes were observed. One possible explanation for this result could be the weighted use of extreme marginal organs in these high risky recipients. Indeed, after the earliest months from ULT introduction, when the policy was to transplant the first compatible organ, we tried to optimize the donor-recipient match. This prudential approach might have prolonged the time spent on the WL, with augmented death risk due to worsening of clinical conditions that, combined with the shortage of donors, in this study is higher $(19.3 \%)$ than our previous (5.8\%) and national (11\%) data (4).

Considering recipient pre-operative risk factors, we found that VA ECMO presence and length were related to mortality, and this could be associated to a severe impairment of the patient that needs both respiratory and haemodynamic support. Notably, all the patients bridged with VA ECMO presented with secondary $\mathrm{PH}$, confirming this condition as an additional organ failure leading to a worse outcome. Our experience on such patients is consistent with previous findings of VA ECMO as useful while waiting for LT, regardless the source of $\mathrm{PH}$. As stated, VA ECMO resulted in an immediate increase of mortality risk, probably because this represented a sort of no-return point, beyond which the patient could only benefit from the transplant as soon as possible. This confirms what previous studies pointed out: recently, Grimm identified preoperative ECMO and ICU admission as risk factors for 1-year mortality (23) and Mulvihill found those factors predictive for postoperative ECMO, directly related to reduced 6-months survival (24). Moreover, preoperative ECMO and $\mathrm{MV}$ are known as individual risk factors for reduced 1 -year survival $(25,26)$. Since all of our patients were admitted to ICU and supported with ECMO or MV prior to transplantation, this could explain the slightly higher in-hospital mortality, when compared to other Italian and foreign elective LT series (27). In addition, our findings demonstrated a relationship between preoperative CRP levels and post-transplant mortality, thus confirming this biochemical marker as useful when assessing postoperative course of a patient (28). Indeed, CRP is involved in any kind of inflammation, not only in that of infectious etiology. In our cases, high levels of CRP were probably not related to infection, since we did not find other parameters, like 
procalcitonin or infection itself, as postoperative mortality risk factors.

Finally, we tried to identify a cut-off LAS value beyond which LT loses its benefits, in terms of increased posttransplant mortality: the analysis demonstrated a higher mortality when LAS increases over 90 units. Previous paper found a 1.5-2-fold greater risk of death for patients with LAS higher than 60 (16), while Russo found this limit at 75 (15). A more recent study (29) only reported higher mortality with higher LAS, without a determination of cutoff value and confirming the ability of such score to allow quick access to transplantation. Notably, when using LAS to define WL priorities, patients with lower probability of post-transplant survival receive lower LAS, thus reducing their possibility to be transplanted (6). Since LAS is not used in Italy, probably we transplanted patients that, in a country that adopts LAS, would not have received LT. On the other hand, only 4 patients in our study had LAS $>90$, thus maybe confounding statistical results.

The main limitation of this study is the lack of a matched control group. Indeed, we preferred the comparison with the recent literature production. We also know that the population is still small, but on the other hand involves a particular group of patients in a country with LT activity limited to about $120-140$ per year.

In conclusion, this multicentre report concerning the Italian Urgent Lung Transplant program demonstrates that ULT is feasible and, despite higher in-hospital mortality, mid-term survival is comparable to other national data. Proper recipient selection, involving multidisciplinary team discussion, seems to be mandatory to gain the maximum advantage from ULT.

\section{Acknowledgments}

None.

\section{Footnote}

Conflicts of Interest: The authors have no conflicts of interest to declare.

Ethical Statement: The authors are accountable for all aspects of the work in ensuring that questions related to the accuracy or integrity of any part of the work are appropriately investigated and resolved. This study was approved by every single Institutional review board (protocol number 0027736) and performed in accordance with accepted ethical standards. Informed consent was obtained before using patients' data.

\section{References}

1. Kotecha S, Hobson J, Fuller J, et al. Continued Successful Evolution of Extended Criteria Donor Lungs for Transplantation. Ann Thorac Surg 2017;104:1702-9.

2. Roux A, Beaumont-Azuar L, Hamid AM, et al. High emergency lung transplantation: dramatic decrease of WL mortality death rate without relevant higher posttransplant mortality. Transpl Int 2015;28:1092-101.

3. Egan TM, Edwards LB. Effect of the lung allocation score on lung transplantation in the United States. J Heart Lung Transplant 2016;35:433-9.

4. Boffini $M$, Venuta F, Rea F, et al. Urgent lung transplant programme in Italy: analysis of the first 14 months. Interact Cardiovasc Thorac Surg 2014;19:795-800.

5. Schiavon M, Faggi G, Di Gregorio G, et al. Single-center experience in urgent lung transplantation program in a country with a shortage of donors: Does the end justify the means? Clin Transplant 2017. doi: 10.1111/ctr.13129.

6. Egan TM, Murray S, Bustami RT, et al. Development of the new lung allocation score in the United States. Am J Transplant 2006;6:1212-27.

7. Oto T, Levvey BJ, Whitford H, et al. Feasibility and utility of a lung donnor score: correlation with early posttransplant outcomes. Ann Thorac Surg 2007;83:257-63.

8. Galiè N, Humbert M, Vachiery JL, et al. 2015 ESC/ERS Guidelines for the diagnosis and treatment of pulmonary hypertension. Eur Respir J 2015;46:903-75.

9. Christie JD, Bellamy S, Ware LB, et al. Construct validity of the definition of primary graft dysfunction after lung transplantation. J Heart Lung Transplant 2010;29:1231-9.

10. Stewart S, Fishbein MC, Snell GI, et al. Revision of the 1996 working formulation for the standardization of nomenclature in the diagnosis of lung rejection. J Heart Lung Transplant 2007;26:1229-42.

11. Durrleman S, Simon R. Flexible regression models with cubic splines. Stat Med 1989;8:551-61.

12. R Development Core Team. R: A language and environment for statistical computing. [Internet]. Vienna, Austria: R Foundation for Statistical Computing; 2015. Available online: http://www.R-project.org

13. Harrell FEJ. rms: Regression Modeling Strategies. R package version 4.1-3. 2014; Available online: http:// CRAN.R-project.org/package $=$ rms

14. Shafii AE, Mason DP, Brown CR, et al. Too high for 
transplantation? Single-center analysis of the lung allocation score. Ann Thorac Surg 2014;98:1730-6.

15. Russo MJ, Iribarne A, Hong KN, et al. High lung allocation score is associated with increased morbidity and mortality following transplantation. Chest 2010;137:651-7.

16. Liu V, Zamora MR, Dhillon GS, et al. Increasing lung allocation score predict worsened survival among lung transplant recipients. Am J Transplant 2010;10:915-20.

17. Verleden GM, Dupont L, Yserbyt J, et al. Recipient selection process and listing for lung transplantation. J Thorac Dis 2017;9:3372-84.

18. Orsini B, Sage E, Olland A, et al. High-emergency waiting list for lung transplantation: early results of a nation-based study. Eur J Cardiothorac Surg 2014;46:e41-7.

19. Zych B, Garcia Sàez D, Sabashnikov A, et al. Lung transplantation from donors outside standard acceptability criteria: are they really marginal? Transpl Int 2014;27:1183-91.

20. Snell GI, Paraskeva M, Westall GP. Donor selection and management. Semin Respir Crit Care Med 2013;34:361-70.

21. Mulligan MJ, Sanchez PG, Evans CF, et al. The use of extended criteria donors decreases one-year survival in high-risk lung recipients: a review of the United Network of Organ Sharing Database. J Thorac Cardiovasc Surg 2016;152:891-898.e2.

22. Sommer W, Kuhn C, Tudorache I, et al. Extended criteria donor lungs and clinical outcome: results of an alternative allocation algorithm. J Heart Lung Transplant 2013;32:1065-72.

Cite this article as: Schiavon M, Faggi G, Rosso L, Luzzi L, Comacchio GM, Gregori D, Nosotti M, Damarco F, Dell'Amore A, Bennet D, Fossi A, Paladini P, Santambrogio L, Rea F. Outcomes and risk factors identification in urgent lung transplantation: a multicentric study. J Thorac Dis 2019;11(11):4746-4754. doi: 10.21037/jtd.2019.10.55
23. Grimm JC, Valero V, Magruder JT, et al. A novel risk score that incorporates recipient and donor variables to predict 1 -year mortality in the current era of lung transplantation. J Heart Lung Transplant 2015;34:1449-54.

24. Mulvihill MS, Yerokun BA, Davis RP, et al. Extracoporeal membrane oxygenation following lung transplantation: indications and survival. J Heart Lung Transplant 2018;37:259-67.

25. Russo MJ, Davies RR, Hong KN, et al. Who is the high-risk recipient? Predicting mortality after lung transplantation using pretransplant risk factors. J Thorac Cardiovasc Surg 2009;138:1234-1238.e1.

26. Mason DP, Thuita L, Nowicki ER, et al. Should lung transplantation be performed for patients on mechanical respiratory support? the US experience. J Thorac Cardiovasc Surg 2010;139:765-773.e1.

27. Lund LH, Khush KK, Cherikh WS, et al. The Registry of the International Society for Heart and Lung Transplantation. J Heart Lung Transplant 2017;36:1037-46.

28. Min JJ, Nam K, Kim TK, et al. Relationship between early postoperative C-reactive protein elevation and long-term postoperative major adverse cardiovascular and cerebral events in patients undergoing off-pump coronary artery bypass graft surgery: a retrospective study. $\mathrm{Br} \mathrm{J}$ Anaesth 2014;113:391-401.

29. Hayanga JA, Lira A, Vlahu T, et al. Lung transplantation in patients with high lung allocation scores in the US: evidence for the need to evaluate score specific outcomes. J Transplant 2015;2015:836751. 